\title{
Análisis de la incidencia de talleres formativos en el desarrollo de competencias comunicativas en futuros docentes y ambientólogos
}

\author{
Rocío Jiménez-Fontana \\ Departamento de Didáctica, Facultad de Ciencias de la Educación. Universidad de Cádir. Cádir. España \\ rocio.fontana@uca.es \\ Lourdes Aragón \\ Departamento de Didáctica, Facultad de Ciencias de la Educación. Universidad de Cádiæ: Cádiæ: España. \\ lourdes.aragon@uca.es \\ Gemma Albendín (D) \\ Departamento de Toxicología. Facultad de Ciencias del Mary Ambientales. Universidad de Cádiæ. Cádiæ: \\ España.gemma.albendin@uca.es
}

[Recibido: 1 diciembre 2019. Revisado: 16 abril 2020. Aceptado: 9 mayo 2020.]

\begin{abstract}
Resumen: La presente investigación se contextualiza en un Proyecto de Actuaciones Avaladas (Universidad de Cádiz, 2018-2019), con el objeto de organizar talleres formativos para mejorar la competencia comunicativa y creación de pósteres científicos en dos titulaciones universitarias, el Grado en Educación Primaria y el Grado en Ciencias Ambientales. Se analiza cómo incide la realización de dichos talleres en el desarrollo de las habilidades comunicativas escritas y orales, atendiendo a las calificaciones obtenidas. Se emplea como instrumento una rúbrica diseñada por el equipo de docentes. El análisis estadístico muestra que los talleres inciden positivamente en el desarrollo de dichas habilidades.
\end{abstract}

Palabras clave: Competencia Científica; Congresos; Habilidades Comunicativas; Innovación Docente; Talleres Formativos.

Analysis of the invidence of training workshops in the development of comunication skills for future teachers and professionals in environmental science

Abstract: This research is part of a Project of Guaranteed Actions (University of Cádiz, 2018-2019), in order to organize training workshops to improve communicative competence and creation of scientific posters in two university degrees, the Degree in Primary Educationand and the Degree in Environmental Sciences. It analyzes how the performance of these workshops affects the development of written and oral communication skills, based on the qualifications obtained. A rubric designed by the team of teachers is used as an instrument. The statistical analysis shows that the workshops have a positive impact on the development of these skills.

Keywords: Scientific Competence; Congress; Communicative Skills; Teaching Innovation; Training Workshops.

Para citar este artículo: Jiménez-Fontana R., Aragón L., Albendín G. (2020) Análisis de la incidencia de talleres formativos en el desarrollo de competencias comunicativas en futuros docentes y ambientólogos. Revista Eureka sobre Enseñanza y Divulgación de las Ciencias 17(3), 3203. doi: 10.25267/Rev_Eureka_ensen_divulg_cienc.2020.v17.i3.3203

\section{Introducción}

El desarrollo de la competencia científica requiere de prácticas lingüísticas, principalmente por dos motivos. El primero, porque la creación de conocimiento a partir del razonamiento tiene una componente social que requiere comunicación. El segundo, porque el lenguaje permite establecer las relaciones entre conceptos e ideas (Domènech Casal 2019). Como indican Crujeiras y Alexaindre (2012), enseñar ciencias implica no solo aprender modelos e ideas científicas, sino también aspectos sobre la ciencia. Esto es, conocer algunos rasgos de cómo trabaja la ciencia, leer, escribir, discutir, argumentar y, en definitiva, comunicar conocimiento.

\author{
Revista Eureka sobre Enseñanza y Divulgación de las Ciencias \\ Universidad de Cádi:. APAC-Eureka. ISSN: 1697-011X \\ http:/ / dx.doi.org/10.25267/Rev_Eureka_ensen_divulg_cienc.2020.v17.i3.3203 \\ http:/ / reuredc.uca.es
}


Los estudios sobre el desarrollo de habilidades y competencias comunicativas y su importancia para el desempeño profesional han ocupado en los últimos tiempos un lugar importante en los análisis de los círculos científicos (Pompa López y Pérez López 2018). En los futuros profesionales de cualquier disciplina su calidad formativa y preparación, pasa por aprender a desarrollar su comunicación oral y escrita, así como tomar conciencia de sus áreas de mejora y evolución de su propio aprendizaje en el desarrollo de esta competencia (De la Fuente Prieto et al. 2015). Sin embargo, los egresados presentan diversos niveles de dificultades en su capacidad para comunicarse adecuadamente y sobre todo para redactar documentos de carácter científico (Marines, Heredia, Solís y Mena 2014). En la mayoría de carreras universitarias, y en no pocos postgrados, se observa la carencia de actividades concretas de desarrollo de competencias para la comunicación de los resultados científicos (Montejo 2016). De acuerdo a Neira-Piñeiro et al. (2018) la competencia comunicativa es crucial para cualquier profesional, en especial para los futuros docentes. En su desempeño profesional estos deben enfrentarse ante situaciones comunicativas no solo con su alumnado, también con las familias, las instituciones o con otros docentes. En el marco de los futuros docentes existen algunos estudios que explicitan la necesidad de prestar mayor atención a la hora de dotar de conocimientos, técnicas, habilidades, actitudes, contextos y posibilidades para afrontar con éxito situaciones reales de la práctica profesional, de manera que se denota una grave desconexión entre la formación inicial recibida y lo que se necesita después para el desempeño profesional (Domingo et al. 2013). Algunos trabajos muestran diferencias en el rendimiento académico alcanzado por estudiantes tanto del Grado en Educación Infantil como de Primaria, ante un mismo contenido, dependiendo si este se expresa de manera escrita u oral, siendo significativamente menor en la competencia comunicativa oral (Balaguer Fàbregas et al. 2015). Estos resultados nos ofrecen un escenario en el que no todos los egresados de Ciencias de la Educación y que oficialmente han adquirido las competencias profesionales, son capaces de enfrentarse a las situaciones de interacción y comunicación que su profesión requiere (Domingo et al. 2013).

En el caso de estudiantes del Grado en Ciencias Ambientales, un análisis realizado sobre su participación en actividades dirigidas a la adquisición de habilidades comunicativas orales y escritas, mostró diferencias entre las calificaciones obtenidas por los alumnos que no participaron, y aquellos que sí lo hicieron. Asimismo, los estudiantes que participaron valoraron positivamente dicha iniciativa (Albendín et al. 2019). Otros estudios realizados en el marco de estudiantes de la Licenciatura en Ciencias Ambientales, han detectado ciertas deficiencias en sus habilidades orales, patentes a la hora de realizar debates en clase; los estudiantes suelen hablar todos a la vez, exponiendo sus argumentos sin escuchar a sus compañeros, empleando un volumen de voz inadecuado. Por otro lado, los estudiantes más tímidos no suelen participar en este tipo de actividades y la mayoría de ellos emplea un vocabulario excesivamente informal y vulgar (Herrera et al. 2012).

En el caso de los Grados en Educación Primaria (GEP) y Ciencias Ambientales (GCA), contexto al que pertenecen los participantes de este estudio, encontramos que en la Universidad de Cádiz (UCA) las habilidades relacionadas con la competencia científica y, concretamente, las que tienen que ver con la comunicación tanto oral como escrita se encuentran entre las competencias básicas de ambas titulaciones (UCA (2012) y UCA (2013) respectivamente). Así, la competencia CB3 - Que los estudiantes tengan la capacidad de reunir e interpretar datos relevantes (normalmente dentro de su área de estudio) para emitir juicios que incluyan una reflexión sobre temas relevantes de índole social, científica o ética y la CB4 Que los estudiantes puedan transmitir información, ideas, problemas y soluciones a un público tanto especializado como no especializado. Sin embargo, según las valoraciones de los estudiantes en cursos anteriores, ponen de manifiesto tener carencias en dichas competencias, 
concretamente, en la habilidad de comunicación escrita mediante la elaboración del póster y la defensa oral del trabajo (Jiménez-Tenorio y Vicente, 2018). En el caso de estudiantes de Ciencias Ambientales en el marco de un Simposio Científico de Alumnos de Ciencias del Mar y Ambientales (SACMA), los docentes participantes constataron que los alumnos tenían escasa formación en estas mismas competencias. Estas observaciones iniciales fueron las que han motivado la experiencia que aquí se presenta, enmarcada dentro de un Proyecto de Actuaciones Avaladas para la Mejora Docente concedido por el Vicerrectorado de Innovación Docente de la UCA. Este se ha desarrollado durante el primer semestre del curso 2018-2019, y han participado estudiantes del GEP y del GCA. En esta línea, consideramos que es igual de importante desarrollar y poner en marcha procesos educativos innovadores como investigar sobre ellos. Es habitual que en muchos casos se obvie la evaluación o seguimiento respecto al proceso conducente a su implantación en los escenarios educativos (Díaz-Barriga 2010).

\section{El Congreso Científico y los Talleres como estrategias didácticas en el Espacio Europeo de Educación Superior}

Como se indicaba al inicio, la formación de ciudadanos competentes científicamente implica no solo el conocimiento de los principales modelos científicos, sino también adquirir habilidades y estrategias para la construcción del conocimiento científico como la formulación de hipótesis, el diseño de experimentos, el análisis de datos o la comunicación de resultados entre otros (Hodson 1994, Pedrinaci et al 2012). También es fundamental comprender los mecanismos y dinámicas sociales por los que la comunidad científica valida el conocimiento (Llorente et al. 2017).

En los últimos años se han llevado a cabo estrategias didácticas innovadoras que promueven el desarrollo de la competencia comunicativa en el marco de la Educación Superior. Por un lado, se encuentran los talleres. Estos pueden considerarse como unidades productivas de conocimiento a partir de una realidad concreta, para ser transferidos a esa realidad, donde los participantes trabajan haciendo converger teoría y práctica (Betancourt et al. 2011). Se considera que la persona que facilita el taller debe tener ciertas habilidades pedagógicas que le permitan orientar el proceso de los participantes, de manera que se cumpla el objetivo planteado para el taller (Rodríguez y Vargas 2009). Algunas experiencias didácticas como la llevada a cabo por Asensio Pastor (2019) en estudiantes universitarios, indican que los talleres son una metodología adecuada para trabajar la alfabetización en escritura académica, al ser un modelo pedagógico de aprendizaje reflexivo-experimental (Ibermón y Medina 2008). A pesar de obtener valoraciones positivas por parte del alumnado, parece ser que un gran número asiste por sugerencia de los docentes y no tanto por iniciativa personal.

Por otro, se encuentra la organización de Congresos Científicos en diferentes titulaciones con resultados muy satisfactorios en cuanto a la mejora de la competencia comunicativa como en competencias profesionales (González-Sanz y Barquero-González 2012, González et al. 2012). El uso de Congresos Científicos para estudiantes pregraduados es más habitual en titulaciones relacionadas con Ciencias de la Salud. Así, el Congreso Nacional de Investigación en Ciencias de la Salud para Alumnos Pregraduados, organizado por la Universidad Complutense de Madrid, tiene en XIII edición como objetivo fomentar la formación universitaria integral, iniciar al alumnado en el campo de la investigación científica y promover el encuentro entre titulaciones de Ciencias de la Salud (Blanco et al. 2017).

Las potencialidades didácticas de un Congreso cumplen con las características basadas en los doce principios de Rath, usadas por Santos Guerra (1991) para evaluar los materiales curriculares, pues permiten al alumno tomar decisiones razonables y enfrentarse a las consecuencias de su elección, desempeñar un papel activo al investigar, exponer, observar, 
entrevistar, participar en simulaciones., etc. Lo comprometen en la investigación de ideas, en aplicación de procesos intelectuales o problemas personales y sociales; lo implican en la realidad; atienden a diferentes intereses propiciando tareas como imaginar, comparar, clasificar o resumir; estimulan a examinar ideas o aplicar procesos intelectuales en nuevas situaciones, contextos o materias; exigen detenerse en temas en los que habitualmente no lo hacen, centrados en problemas socio ambientales; obligan a aceptar el riesgo, el fracaso y la crítica; exigen que revisen y perfeccionen sus esfuerzos iniciales; los comprometen en la aplicación y dominio de reglas y normas, controlando lo hecho y sometiéndolo a análisis; dan la oportunidad de planificar con otros y participar en el desarrollo y en los resultados y acogen intereses que potencian el compromiso personal (Santos Guerra 1991). Ligado a la realización de Congresos Científicos, el póster posee un valor relevante como formato para comunicar las producciones del alumnado. Existe mucha bibliografía sobre la elaboración y el uso del póster científico como medio para transmitir y publicar los resultados científicos procedentes de investigaciones (Berbey-Álvarez et al. 2017). Son una potente herramienta para estructurar razonamientos que permite al alumnado escribir y pensar de forma análoga a como se escribe y se piensa la ciencia (Domènech Casal 2019), que contribuyen a su formación por el control metacognitivo que demandan y por reproducir la interacción entre el investigador y la comunidad científica (Montejo 2016). Como recurso para la docencia posee gran relevancia, dado que diseñar un póster puede ser un proceso complejo, en el que se ponen en juego destrezas como sintetizar, organizar, analizar y presentar de manera concisa la información que se desea comunicar (Díaz y Muñoz 2013).

\section{Metodología de la Investigación}

\section{Contexto}

Teniendo en cuenta los presupuestos de partida en relación con estrategias didácticas que promueven la habilidad comunicativa, se optó por ofertar dos talleres (T.PC y T.HC) como complemento formativo fuera del horario curricular, y no obligatorios. Dichas estrategias didácticas (Asensio Pastor 2019) tienen versatilidad y autonomía al crear estructuras de diálogo y participación entre los diferentes participantes.

Taller de iniciación a la elaboración de pósteres científicos (T.PC)

Este fue impartido por personal técnico de la biblioteca de la UCA. Se ofertó dentro de los programas formativos de la categoría de "Creación, gestión y comunicación de la información". Los objetivos de este taller, así como los contenidos tratados se muestran en la tabla 1.

\begin{tabular}{|c|c|}
\hline \multicolumn{2}{|c|}{$\begin{array}{l}\text { Tabla 1. Características del taller de formación “iniciación a la elaboración de pósteres científicos". Fuente: } \\
\text { Elaboración propia }\end{array}$} \\
\hline CARACTERÍSTICAS & INICIACIÓN A LA ELABORACIÓN DE PÓSTERES CIENTÍFICOS \\
\hline Objetivos & $\begin{array}{l}\text { Adquirir y desarrollar conocimientos básicos de cómo elaborar un póster } \\
\text { académico }\end{array}$ \\
\hline \multirow{5}{*}{ Contenidos } & $\begin{array}{l}\text { Aprender los elementos indispensables que conforman el póster } \\
\text { académico, así como su disposición y jerarquización en lo que al diseño se } \\
\text { refiere }\end{array}$ \\
\hline & El póster científico: concepto, ventajas, tipos \\
\hline & Estructura del contenido \\
\hline & Diseño: distribución de elementos, colores, texto, elementos gráficos \\
\hline & Software \\
\hline
\end{tabular}


El taller se desarrolló en una sola sesión, con una duración total de cuatro horas, divididos en una primera parte teórica donde se desarrollaban los contenidos del mismo y una segunda parte práctica destinada a la elaboración del primer póster científico de los estudiantes. Para ello se hizo uso de las herramientas propuestas en el taller y de un artículo proporcionado por la formadora. Para este taller se programaron tres sesiones diferentes, el 26 de noviembre, el 4 y 17 de diciembre de 2018 en horario extraescolar de 16:00 a 20:00 horas. Los alumnos en función de su disponibilidad podían elegir uno de los tres días propuestos, respetando siempre el límite de aforo. En este taller participaron un total de 54 estudiantes, 40 del GEP y 14 del GCA.

\section{Taller de formación en habilidades comunicativas (T.HC)}

El taller de "formación en habilidades comunicativas" fue impartido por un experto en Coaching, responsable de varios cursos de formación relacionados con la comunicación y dirigidos al profesorado de la UCA. El objetivo y contenidos de este taller se exponen en la tabla 2. El taller tuvo una duración de cinco horas, con un carácter eminentemente práctico en el que se fomentó la participación de los alumnos para hacerles superar sus reticencias a hablar en público y remarcar la importancia que tiene la comunicación para ellos y para su futura labor como docentes y futuros profesionales (Tabla 2). Se organizaron dos sesiones para este taller, el 10 y 17 de diciembre en horario extraescolar de 16:00 a 21:00 horas, y al igual que en el taller de elaboración de póster científicos, los alumnos podían elegir libremente a qué sesión querían asistir. En este taller participaron un total de 36 estudiantes, 26 del GEP y 10 del GCA.

\begin{tabular}{ll}
\hline Tabla 2. Características del taller "formación en habilidades comunicativas". Fuente: Elaboración propia \\
\hline Características & FORMACIÓN EN HABILIDADES COMUNICATIVAS \\
Objetivos & Presentar claves para mejorar la comunicación oral \\
Contenidos & La planificación y estructura del discurso \\
& Diseño, exposición y uso del lenguaje verbal y no verbal
\end{tabular}

\section{Congreso II MAFEPRID-CN2019}

Se planificó y celebró una simulación de Congreso Científico en el que los estudiantes, en el papel de investigadores, elaboraron sus propias comunicaciones, presentando sus pósteres y defendiendo sus trabajos. De esta manera se trabajaron habilidades orales y escritas, desarrollando la comunicación y argumentación científica. En el mismo debían poner en juego los aprendizajes adquiridos durante la asignatura y, especialmente, en ambos talleres. Para el congreso, el equipo docente, diseñó y elaboró cartelería para difundir el evento, se publicó un anuncio en la web de la UCA para darle visibilidad, se elaboró un reglamento de participación y publicación y un programa de sesiones, e impresión de un díptico informativo y libro de actas. Además, como suelen hacer algunos congresos y para incentivar la motivación del alumnado, se organizó un concurso para seleccionar los mejores trabajos presentados.

El Congreso tuvo lugar del 14 al 18 de enero de 2019 en la Facultad de Ciencias de la Educación. Para su desarrollo se programó una sesión de recogida de documentación y colocación de póster por parte de los alumnos, ocho sesiones de exposición y presentación de trabajos de una hora y media cada una en las que los participantes tuvieron la oportunidad de divulgar e intercambiar los resultados de sus investigaciones y una última sesión de entrega de premios. En las sesiones de exposición cada grupo tuvo que presentar y defender durante 10 minutos sus trabajos ante un Comité Científico formado por personal docente e investigador del Área de Didáctica de Ciencias Experimentales. Dicho Comité eligió las mejores comunicaciones y defensas realizadas para cada grupo. Los segundos y terceros premios 
fueron dados por los propios alumnos. De este modo, fueron premiados nueve de los cuarenta trabajos presentados en el congreso, siguiendo los criterios de la rúbrica elaborada para tal fin.

\section{Actividad voluntaria: Bloques temáticos sobre la toxicología de contaminantes relevantes}

En cuanto al GCA, se realizó en la asignatura de Toxicología Ambiental y Salud Pública. Esta posee un extenso temario, difícil de abarcar. Además, los docentes del Área de Toxicología están muy involucrados en la organización del SACMA, por lo que son conscientes de las deficiencias en la formación de los estudiantes en estas habilidades. Por estos motivos, se decidió participar en este Proyecto, mediante la realización de una actividad de trabajo en grupo, en la que los alumnos elegían un tema de los cuatro propuestos (Toxicidad de diferentes familias de compuestos como hidrocarburos aromáticos policíclicos, metales, plaguicidas y dioxinas). Los alumnos realizaban una búsqueda de información siguiendo un pequeño guion facilitado por los docentes. Dicha información debían presentarla mediante una comunicación tipo póster. Además, los alumnos debían de realizar una exposición y defensa de su trabajo, y contestar a las preguntas formuladas por los docentes.

\section{Participantes}

Por un lado, la propuesta educativa se realizó en la asignatura de Didáctica de las Ciencias de la Naturaleza I, llevándose a cabo en los tres grupos-clase del $3^{\circ}$ curso del GEP. De manera paralela, la propuesta se llevó a cabo en la asignatura de Toxicología Ambiental, que se imparte en el $4^{\circ}$ curso del GCA. Ambas asignaturas son de 6 créditos ECTS y son del primer semestre. En este estudio han participado un total de 207 estudiantes. Al GEP pertenecen 171 estudiantes, 46 hombres y 125 mujeres, de edades comprendidas entre 20 y 23 años. En el caso del GCA, son 36 estudiantes, 17 hombres y 19 mujeres, de entre 21 y 23 años de edad. Para la elaboración y creación de los pósteres, los estudiantes se agruparon en equipos de trabajo de 5-6 componentes cada uno. Para el GEP, se formaron un total de 39 equipos; en 13 de ellos, al menos uno de sus integrantes asistió al T.PC, y en 26 equipos no asistió ningún componente al taller. En el caso de los T.HC, en 10 equipos, al menos un componente, asistió al taller, y en 29 no asistió ningún componente. En el GCA, se formaron 9 equipos de 3-5 componentes cada uno. En este caso, en 3 equipos, al menos algún integrante asistió al T.PC, y 6 equipos no asistieron. De manera similar, para el T.HC, 3 equipos asistieron y 6 no asistieron. En la experiencia se implicaron un total de 7 docentes.

\section{Planteamiento del problema de investigación: cuestiones a analizar}

La presente investigación tiene como objetivo principal analizar la incidencia de la realización de talleres para desarrollar habilidades comunicativas y de elaboración de pósteres científicos en estudiantes del GEP durante la simulación de un Congreso Científico, y en el caso de los estudiantes del GCA, durante la exposición de sus trabajos en una actividad voluntaria en la asignatura de Toxicología Ambiental y Salud Pública. Para ello, nos planteamos las siguientes cuestiones objeto de análisis:

1. ¿Existen diferencias significativas entre aquellos estudiantes que han asistido a los talleres y los que no?

2. Para cada taller, ¿en qué aspectos relacionados con la habilidad comunicativa oral y la habilidad comunicativa escrita se producen las mayores diferencias?

3. ¿Existe relación alguna entre el desarrollo de la habilidad comunicativa oral y la habilidad comunicativa escrita? 
4. ¿Existen diferencias significativas en las habilidades comunicativas entre estudiantes de ambos Grados, enmarcados en diferentes Áreas de Conocimiento (Sociales/Experimentales)?

\section{Instrumentos de recogida de información}

Las habilidades comunicativas orales y escritas fueron puestas en juego y evaluadas en dos actividades diferentes. En el caso del GCA, los estudiantes expusieron y defendieron sus pósteres, en horario curricular. Los trabajos fueron evaluados tanto por los docentes del Área de Toxicología como por los propios compañeros. Asimismo, los estudiantes evaluaron su participación y la de sus compañeros dentro de su equipo de trabajo. En el caso de GEP, el eje de la experiencia fue configurado por la participación en la simulación de un Congreso Científico, considerada adecuada por muchos estudios para promover la competencia científica (González-Sanz y Barquero-González 2012), especialmente para habilidades de comunicación oral y escrita. No solo tuvieron que defender sus pósteres ante el comité científico y el resto de estudiantes, sino que también llevaron a cabo actividades de coevaluación con ayuda de una rúbrica con el objetivo de promover una lectura atenta y evaluación de los pósteres de los compañeros y reproducir una dinámica real en los congresos científicos, la de observar las comunicaciones con el propósito de vincular los datos y conclusiones de distintos autores a un campo o tema específico y transversal (Llorente et al. 2017).

Así, la experiencia conlleva dos niveles didácticos: un "nivel estructurado" de trabajo en pequeños equipos de investigación alrededor de un conflicto fuertemente anclado a los contenidos y habilidades disciplinarias y un "nivel abierto" de trabajo alrededor de un evento o producto colectivo, centradas en las habilidades de comunicación oral y elaboración de póster científicos.

La rúbrica fue diseñada por tres expertos del Área de Didáctica de las Ciencias Experimentales y uno del Área de Toxicología. De las cuatro dimensiones que recoge, las tres primeras reflejan aspectos de la comunicación escrita y la cuarta dimensión se relaciona con aspectos de comunicación oral. Además, cada dimensión cuenta con indicadores que la caracterizan y descriptores que jerarquizan los niveles. Las puntuaciones que pueden alcanzar los descriptores van de 0 a 4. Así, para la Dimensión I: "Organización de la información", los indicadores se relacionan con la secuencia lógica y la facilidad de seguimiento del discurso escrito. Para la Dimensión II: "Formato de presentación", los indicadores se corresponden con el título y su concreción y precisión; con la inclusión de elementos necesarios como los autores o la afiliación; con la introducción y su utilidad; con la información visual y su equilibrio entre texto y figuras, tablas e imágenes o con la fuente utilizada en cuanto a tipo, tamaño y uso de negritas y cursivas. En el caso de la Dimensión III: "Diseño", los indicadores hacen referencia al uso inteligente de los colores y a los elementos gráficos incluidos, en cuanto a calidad de las imágenes y su uso. La Dimensión IV: "Presentación"; refleja indicadores asociados a la estructura del discurso oral relacionada con la lógica de la organización; el hilo conductor o el uso de ejemplos adecuados; el tiempo de exposición, adecuado al previamente establecido; el contenido del discurso en cuanto a un discurso explicativo con detalles adicionales; el uso del lenguaje con tecnicismos apropiados, rigor científico, entonación y pausas en el discurso y el uso del lenguaje no verbal, con la gesticulación, la proyección corporal y la mirada.

Los pósteres fueron evaluados y calificados a través de dicha rúbrica en dos fases diferentes: in situ durante la defensa por dos docentes del Área de Didáctica de las Ciencias en el caso del GEP y por dos docentes del área de Toxicología en GCA, utilizando el valor medio obtenido por las calificaciones de ambos revisores. Las dimensiones relacionadas con la comunicación 
escrita fueron analizadas posteriormente por tres de los docentes que actuaron como pares ciegos, de las dos áreas implicadas en el proyecto. Para la calificación final de cada equipo de trabajo, se empleó la siguiente escala de valores: 0-4,99, suspenso; 5-6,99 aprobado; 7-8,99 notable; 9-10,0: sobresaliente.

\section{Análisis de los datos}

Los datos de este trabajo se trataron estadísticamente utilizando varios programas informáticos: SPSS para Windows versión 24 como programa de análisis estadístico, y como hoja de cálculo, EXCEL 2016, programa necesario para la organización, gestión y presentación de los datos.

Se verificó la hipótesis de normalidad de varianzas mediante test de Shapiro-Wilk y para comprobar la homogeneidad de varianzas se utilizó el test de Levene. El nivel de significación para aceptar las hipótesis mediante estos fue $\mathrm{p} \leq 0,05$. Tras constatar que los datos no cumplían con las condiciones de una distribución normal, se utilizaron test no paramétricos para comprobar la existencia de diferencias significativas entre los grupos de estudio.

Para estudiar la relación existente entre el desarrollo de habilidades orales y la elaboración de pósteres científicos, se realizó un análisis de correlación (Correlación de Spearman).

\section{Resultados}

El principal objetivo de este trabajo es analizar la incidencia de la realización de talleres para el desarrollo de habilidades comunicativas orales y la elaboración de pósteres científicos entre los estudiantes de los GEP y GCA, para lo cual se realizó el análisis de los datos recogidos mediante la evaluación del trabajo final, utilizando la rúbrica elaborada al efecto. Para ello, como se ha comentado con anterioridad, se han abordado cuatro preguntas objeto de investigación, que se pasan a analizar a continuación:

1. ¿Existen diferencias significativas entre aquellos estudiantes que han asistido a los talleres y los que no?

Para abordar este objetivo se establecieron cuatro grupos dependiendo de la asistencia a los talleres de algún integrante de cada equipo de trabajo (tabla 3).

Tabla 3: Calificaciones del trabajo final obtenidas por los estudiantes de ambos Grados divididos en función de su asistencia o no a los talleres. Fuente: Elaboración propia

\begin{tabular}{lllll}
\hline Grupos & Denominación & N & Media & SD \\
G1 & No asiste & 30 & 6,84 & 0,95 \\
G3 & Asiste solo T.HC & 2 & 8,14 & 0,20 \\
G4 & Asiste solo T.PC & 5 & 7,63 & 0,52 \\
\hline
\end{tabular}

En la tabla 3 se observa que se obtuvieron calificaciones más elevadas en aquellos grupos en los que alguno de sus integrantes asistió a uno o a los dos los talleres. Por otra parte, si comparamos las calificaciones obtenidas por los grupos G1, G2 y G3, el porcentaje de notables fue muy superior al obtenido por el grupo en el que los integrantes no asistió a ninguno de los talleres (G4), siendo de un $78 \%$ y un $40 \%$, respectivamente. Si bien, en el grupo de no asistentes a los talleres se encuentra un equipo de trabajo con el único sobresaliente otorgado por los evaluadores. Estos resultados aportan evidencias de la utilidad de realizar talleres formativos para desarrollar ciertas habilidades comunicativas. En concreto, las habilidades comunicativas requieren de pautas y aspectos claves que deben ser transmitidos por profesionales. Asimismo, se observa una incidencia positiva también en aquellos alumnos 
que han asistido al taller de elaboración de póster. Estos datos reforzarían nuestra hipótesis inicial de la necesidad detectada en futuros profesionales de ambas áreas de conocimiento en cuanto al desarrollo de sus habilidades comunicativas.

2. Para cada taller, ¿en qué aspectos relacionados con la habilidad comunicativa oral y la habilidad comunicativa escrita se producen las mayores diferencias?

Una vez establecido que los talleres formativos ayudan a promover las habilidades comunicativas escritas y orales, pretendemos plantearnos en cuál de los aspectos de cada habilidad, establecidos en la rúbrica de valoración en forma de indicadores, tiene lugar la mayor incidencia. Para ello, los grupos anteriores se organizan en dos únicos grupos de asistentes y no asistentes a cada uno de los talleres impartidos.

Respecto a los indicadores correspondientes a la habilidad comunicativa escrita, mediante la elaboración de pósteres (Figura 1), en la mayoría de los indicadores, se observan mejores calificaciones en aquellos equipos en los que alguno de los integrantes asistió a los talleres, siendo las diferencias entre los dos grupos estadísticamente significativas en dos de los ocho indicadores: fuente utilizada $(\mathrm{p}=0,007)$ y elementos gráficos $(\mathrm{p}=0,015)$.

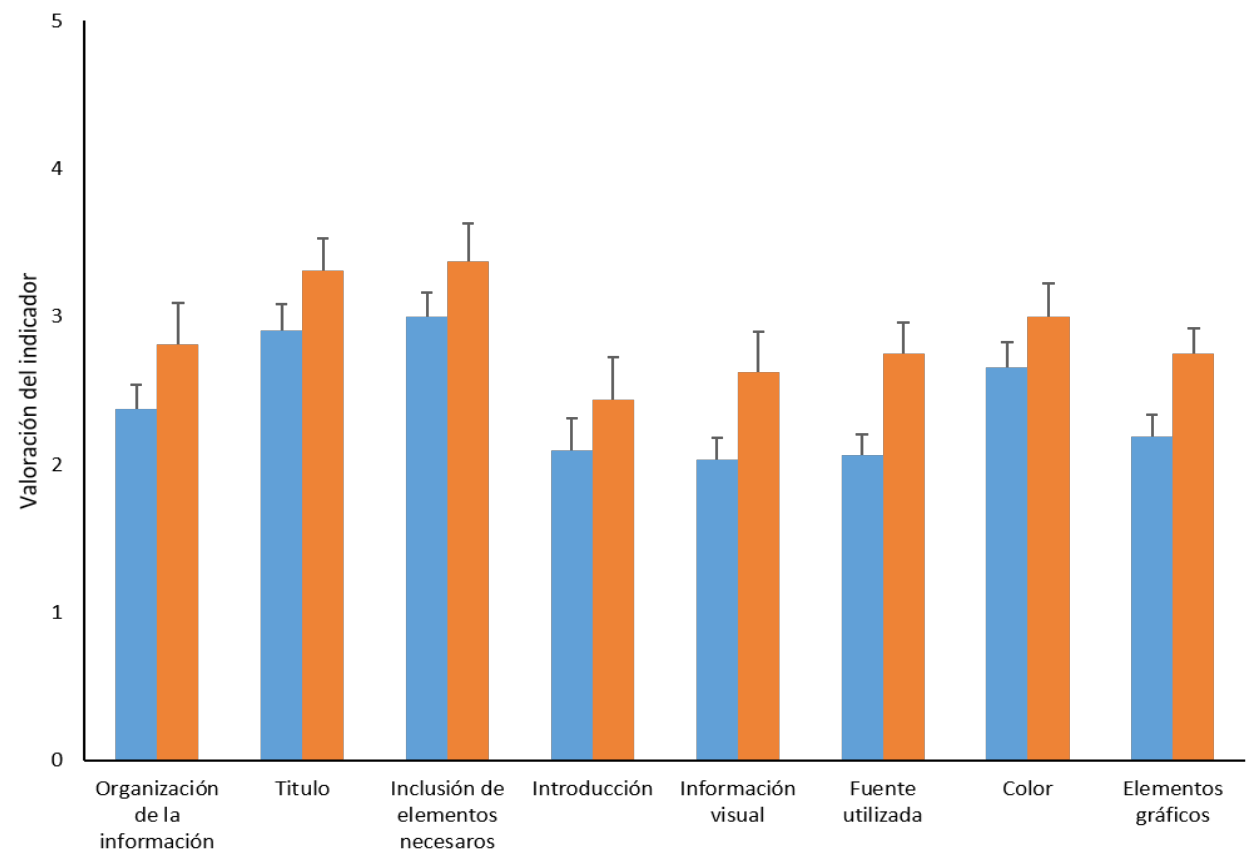

Figura 1: Valoración de los indicadores evaluados para la elaboración de pósteres, no asistente $\mathrm{n}=32$ (azul) y asistente $n=16$ (naranja). Datos representados como media y el error estándar de la media.

Por otra parte, en los indicadores relativos a la habilidad comunicativa oral (Figura 2), no se obtuvieron diferencias estadísticamente significativas. No obstante, en el indicador uso del lenguaje se aprecia una de las mayores diferencias entre los que asisten o no. 


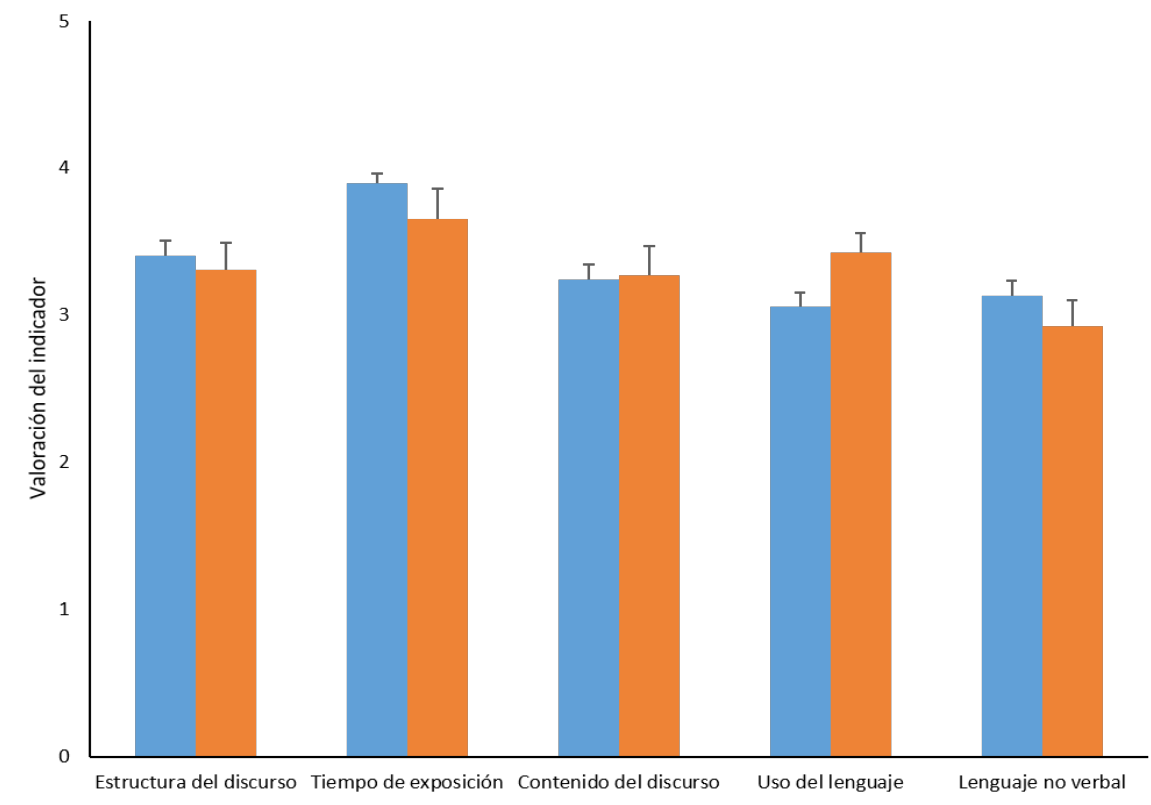

Figura 2: Valoración de los indicadores evaluados para las habilidades de comunicación oral, no asistente $\mathrm{n}=35$ (azul) y asistente $\mathrm{n}=13$ (naranja). Datos representados como media y el error estándar de la media.

3. ¿Existe relación alguna entre el desarrollo de la habilidad comunicativa oral y la habilidad comunicativa escrita?

A priori no parece que el aprendizaje de ambas habilidades vaya en consonancia, o que adquirir unas ayuden a la consecución de las otras, y viceversa. Según los resultados obtenidos, no existe una correlación entre la habilidad comunicativa escrita mediante la elaboración de póster científico y la habilidad comunicativa oral, mediante su defensa $(\mathrm{p}=0,882$; correlación de Spearman). Sin embargo, cuando entramos a correlacionar de manera pormenorizada los aspectos relacionados con ambas habilidades (Tablas 4 y 5), se observa una relación entre el uso de los elementos gráficos (habilidad comunicativa escrita) y el uso del lenguaje verbal (habilidad comunicativa oral).

Además, se aprecia cómo determinados aspectos de la habilidad concreta, escrita u oral, sí establecen correlación con otros de la misma de manera que, al parecer, evolucionan de forma paralela. Para la habilidad comunicativa escrita, sería el caso de aquellos equipos que demuestran una mayor capacidad para organizar bien la información, son capaces al mismo tiempo de realizar una introducción adecuada y usar de forma conveniente los elementos gráficos (Tabla 4).

Tabla 4: Matriz de correlaciones de Spearman $(\mathrm{N}=48)$ para la Habilidad Comunicativa Escrita. * Coeficientes de correlación significativos $(<0,05)$; $* *$ Coeficientes de correlación significativos $(<0,01)$

\begin{tabular}{|c|c|c|c|c|c|c|c|}
\hline & Organización & Titulo & Inclusión & Introducción & $\begin{array}{c}\text { Información } \\
\text { visual }\end{array}$ & Color & $\begin{array}{l}\text { Elementos } \\
\text { gráficos }\end{array}$ \\
\hline Inclusión & & $0,466^{* *}$ & & & & & \\
\hline Introducción & $0,293^{*}$ & $0,501^{* *}$ & $0,335^{*}$ & & & & \\
\hline $\begin{array}{l}\text { Información } \\
\text { visual }\end{array}$ & $0,346^{*}$ & $0,433^{* *}$ & & $0,383^{* *}$ & & & \\
\hline Color & & $0,285^{*}$ & & & & & \\
\hline $\begin{array}{l}\text { Elementos } \\
\text { gráficos }\end{array}$ & $0,357^{*}$ & $0,449^{* *}$ & $0,361^{*}$ & & $0,492^{* *}$ & $0,396^{* *}$ & \\
\hline
\end{tabular}


Para el caso de la habilidad comunicativa oral, los grupos que estructuran bien su discurso, suelen ajustarse al tiempo, seleccionan adecuadamente el contenido a tratar y usan bien el lenguaje verbal y no verbal (Tabla 5).

Tabla 5: Matriz de correlaciones de Spearman $(\mathrm{N}=48)$ para la Habilidad Comunicativa Oral. $*$ Coeficientes de correlación significativos $(<0,05) ;{ }^{* *}$ Coeficientes de correlación significativos $(<0,01)$

\begin{tabular}{lcccc}
\hline & Estructura & Tiempo & Contenido & Uso del lenguaje \\
Tiempo & $0,323^{*}$ & & & \\
Contenido & $0,732^{* *}$ & & & \\
Uso del lenguaje & $0,482^{* *}$ & & $0,425^{* *}$ & $0,551^{* *}$ \\
Lenguaje no verbal & $0,610^{* *}$ & $0,347^{*}$ & $0,427^{* *}$ & \\
\hline
\end{tabular}

4. ¿Existen diferencias significativas en las habilidades comunicativas estudiadas entre estudiantes de ambos Grados, enmarcados en diferentes áreas de conocimiento (Sociales/Experimentales)?

En las figuras $3 \mathrm{a}$ y $3 \mathrm{~b}$ se muestra las medias de las calificaciones obtenidas por los estudiantes de los GEP y GCA en las habilidades comunicativas orales y escritas, respectivamente. El análisis de los datos mostró que no existen diferencias estadísticamente significativas en las calificaciones obtenidas en las habilidades de comunicación escrita por los estudiantes $(p=0,66)$, ni en el caso de las habilidades orales $(p=0,22)$. En el caso de las habilidades comunicativas escritas, asociadas a la elaboración de un póster científico, los valores medios obtenidos fueron en ambos grados muy similares. No obstante, aunque no existen diferencias significativas en cuanto a las calificaciones finales obtenidas en las habilidades comunicativas orales, se observa que los estudiantes del GEP obtuvieron una mejor calificación.

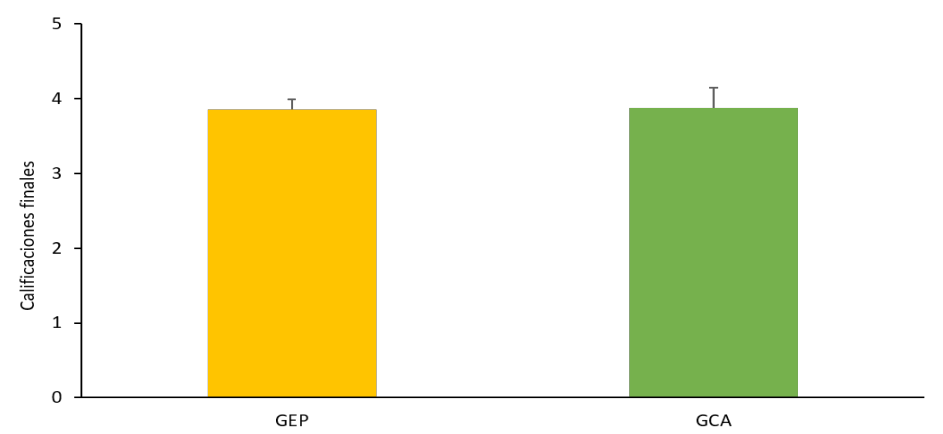

Figura 3a: Calificaciones finales obtenidas por los estudiantes de los Grados participantes en el proyecto para las habilidades escritas. Datos representados como media y el error estándar de la media.

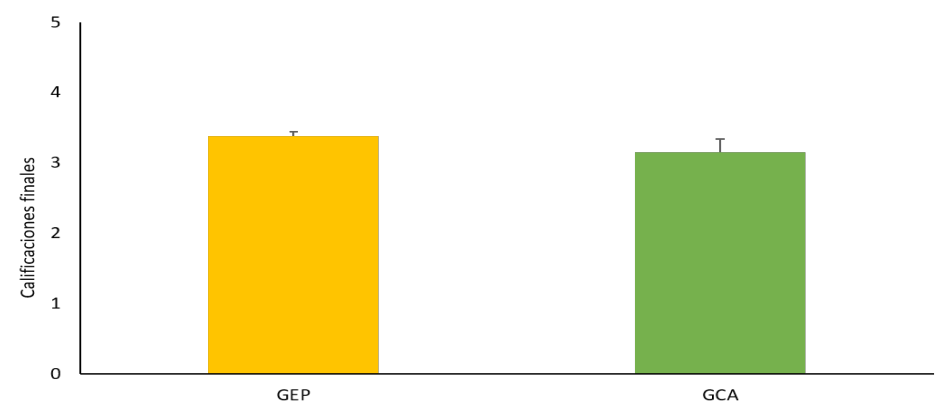

Figura 3b: Calificaciones finales obtenidas por los estudiantes de los Grados participantes en el proyecto para las habilidades de comunicación oral. Datos representados como media y el error estándar de la media. 
Por otro lado, se analizaron los datos obtenidos para ver si había diferencias entre los indicadores evaluados en función de la procedencia de los estudiantes. Es decir, si el Grado que cursaban tenía incidencia sobre alguno de los indicadores objeto de estudio. Para simplificar el análisis se compararon los asistentes y no asistentes de ambos Grados a cada uno de los talleres, estudiándose, por separado, los indicadores específicos de cada taller. En la figura 4, se representan los valores de los indicadores de los grupos de trabajo cuyos integrantes asistieron al T.PC de ambos Grados. Mientras que en la figura 5, se muestran la valoración de los indiciadores de los grupos de trabajo de los estudiantes que no asistieron a este taller. En los dos casos estudiados, no se encontraron diferencias estadísticamente significativas $(\mathrm{p}>0,05)$ entre los estudiantes de ambos grados, tanto los que asistieron al taller (Figura 4) como los que no (Figura 5).

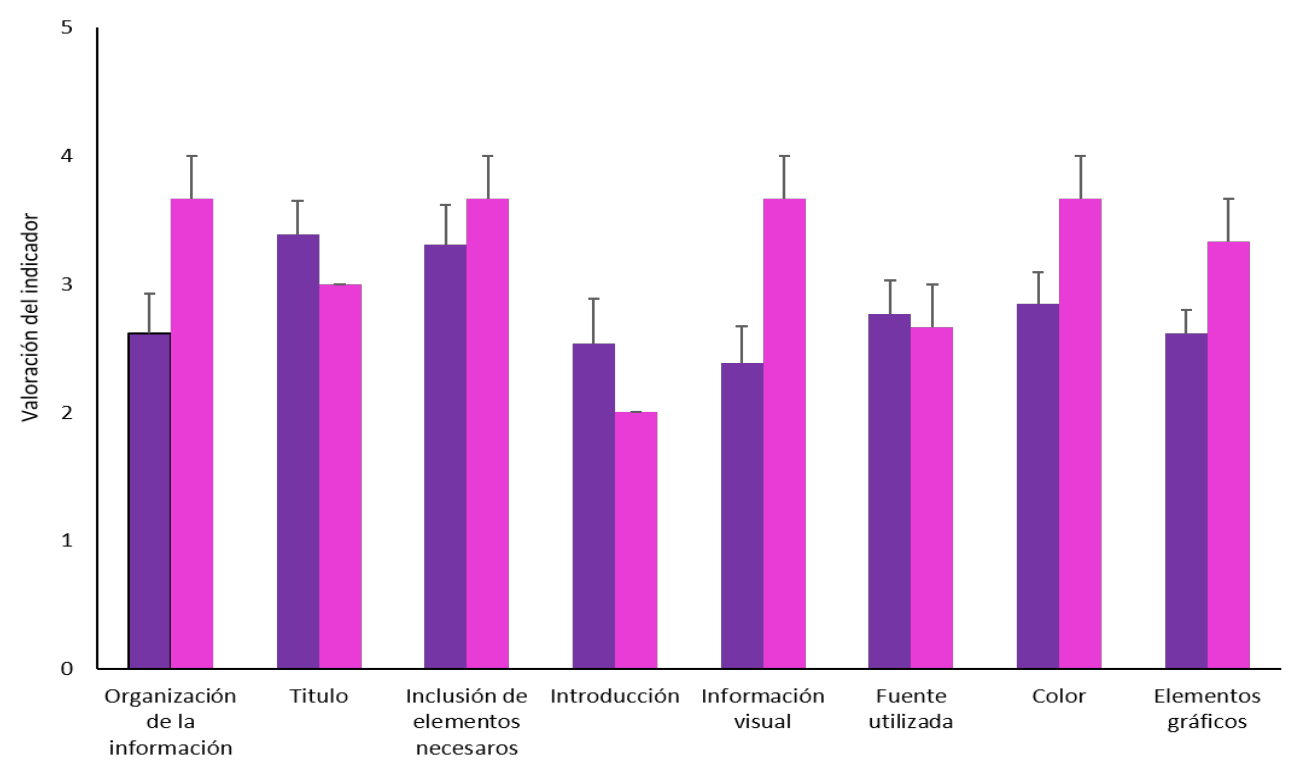

Figura 4: Valoración de los indicadores específicos del taller de pósteres en los grupos de estudiantes que asistieron a este taller según el Grado que cursan. En este caso se ha agrupado en GEP n=13 (morado) y GCA $\mathrm{n}=3$ (rosa). Datos representados como media y el error estándar de la media.

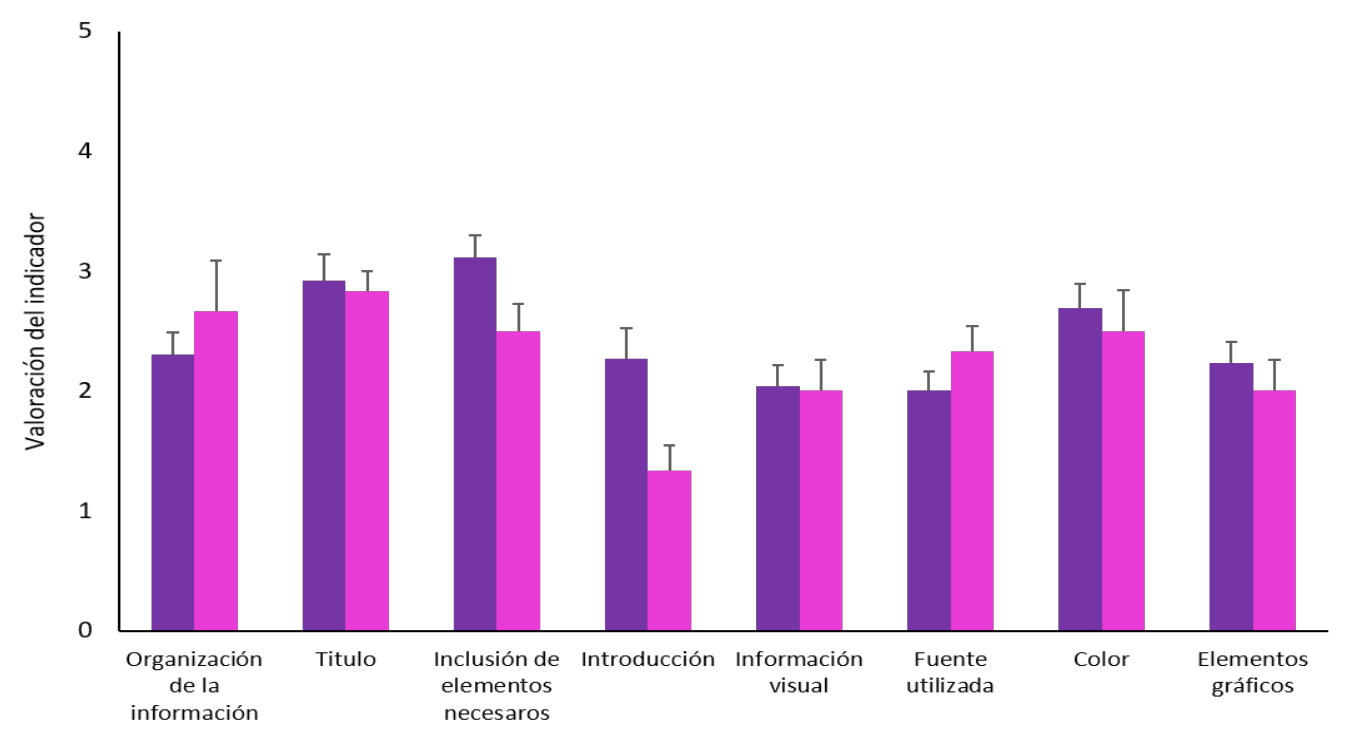

Figura 5: Valoración de los indicadores específicos del taller de pósteres en los grupos de estudiantes que no asistieron a este taller según el Grado que cursan. En este caso se ha agrupado en GEP n=26 (morado) y GCA $\mathrm{n}=6$ (rosa). Datos representados como media y el error estándar de la media. 
El mismo procedimiento se siguió para comparar los equipos de trabajo que asistieron al T.HC (Figura 6) de ambos Grados, y aquellos equipos de trabajo que no asistieron a dicho taller (Figura 7). En la figura 6 se observa una igualdad en la valoración de los indicadores entre los equipos de trabajo de ambos Grados. Sin embargo, en la figura 7 se observa una mayor diferencia en la valoración de los indiciadores específicos del taller entre los estudiantes del GEP y los del GCA.

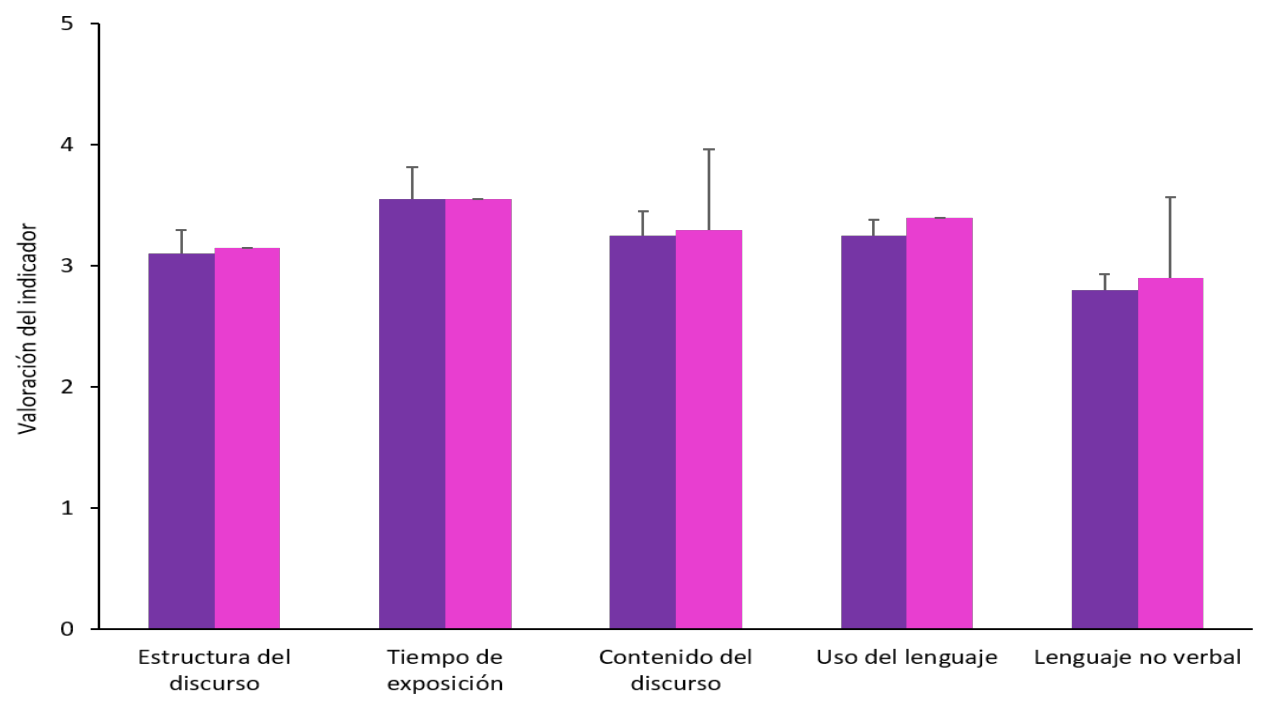

Figura 6: Valoración de los indicadores específicos del taller de comunicación oral en los grupos de estudiantes que asistieron a este taller según el Grado que cursan. En este caso se ha agrupado en GEP n=10 (morado) y GCA $n=3$ (rosa). Datos representados como media y el error estándar de la media.

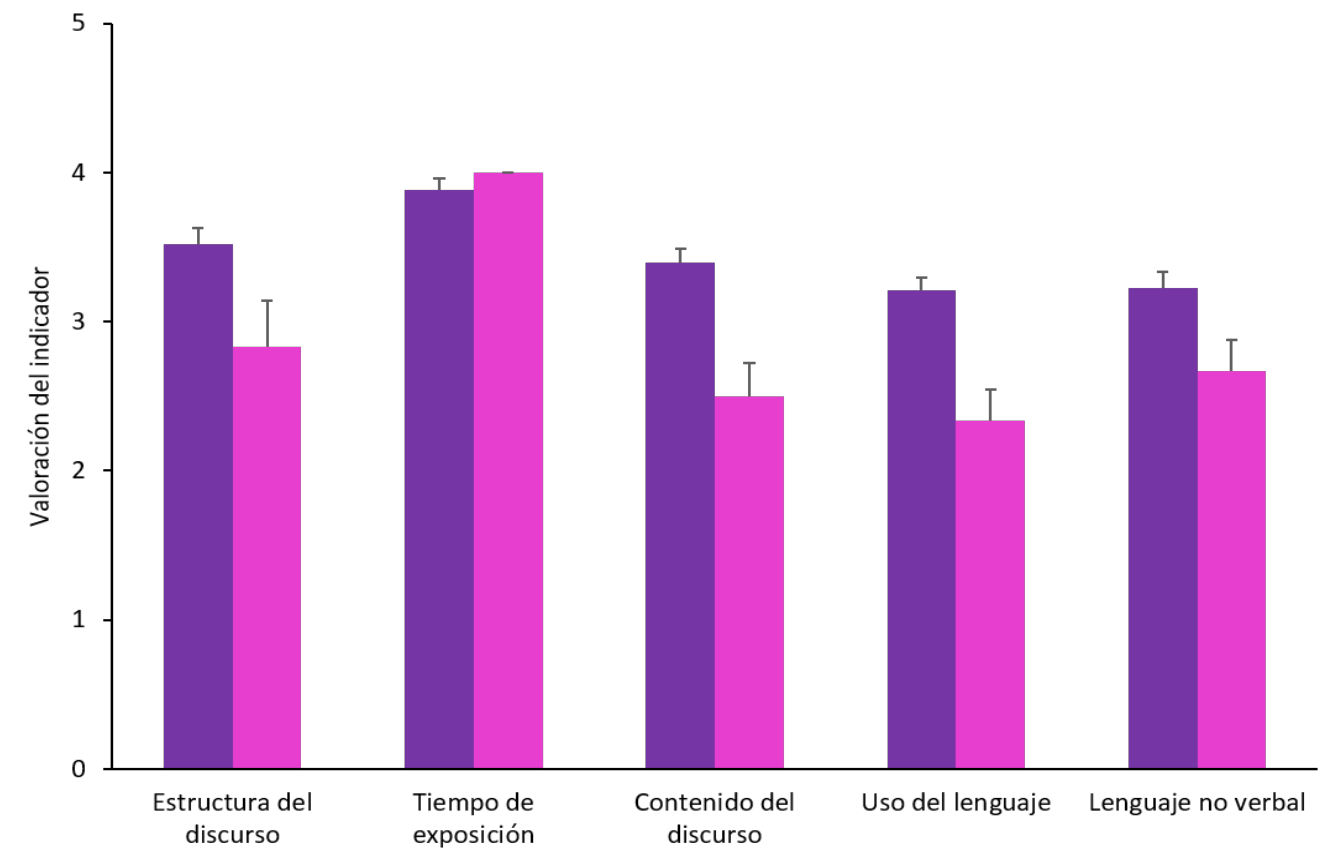

Figura 7: Valoración de los indicadores específicos del taller de comunicación oral en los grupos de estudiantes que no asistieron a este taller según el Grado que cursan. En este caso se ha agrupado en GEP n=29 (morado) y GCA $n=6$ (rosa). Datos representados como media y el error estándar de la media. 


\section{Discusión y Conclusiones}

Los resultados obtenidos en el presente estudio constatan el interés y la relevancia formativa que puede llegar a tener el uso de acciones formativas específicas para desarrollar la competencia comunicativa en el contexto de la Educación Superior, en este caso, con el uso de talleres. Desde una perspectiva crítica es preciso someter cualquier recurso didáctico a un profundo análisis (Moreno 2004). Para ello es fundamental conocer las habilidades del alumnado, identificando sus fortalezas y debilidades (Rodríguez-García y Martínez-Heredia 2019).

En general, como primera conclusión, podemos considerar que los estudiantes de ambos Grados han obtenido calificaciones elevadas. Asimismo, para aquellos que han podido asistir a uno o ambos talleres, han obtenido mejores resultados y, por tanto, ha contribuido de manera satisfactoria al desarrollo de la competencia comunicativa. En términos de indicadores, los estudiantes han sido capaces de diseñar, elaborar y presentar los resultados de sus investigaciones en la simulación de un Congreso, con la creación y defensa de pósteres científicos. Por otro lado, una segunda conclusión, es que de acuerdo a los valores de los indicadores para cada habilidad obtenidos en este estudio, se evidencian mejores resultados para la habilidad comunicativa escrita en comparación con la oral, entre los grupos que asisten frente a los no. Se hace patente así, que existen habilidades comunicativas que requieren tiempo, dedicación y una formación más permanente. En este sentido, estudios como el realizado por Del Barrio et al. (2009) constatan que mejorar las competencias comunicativas, tanto de alumnos como docentes, es un proceso largo y complejo, a la vez que indispensable para cualquier profesional que emplee la comunicación y el lenguaje en su actividad laboral, docente, social o científica. Para ello, fundamentalmente se requiere de la ayuda de expertos y tiempo. Exponen que el hecho de hacer cursos o formación aislada sobre voz, control de ansiedad, del lenguaje, no suele ser eficaz y establecen que los mejores resultados se obtienen tras construir un marco teórico sólido, y después de mucha práctica. Por tanto, existe una propuesta de mejora vital, a la hora de formar a futuros profesionales, docentes o ambientólogos, y es incidir de manera explícita en el desarrollo de la competencia comunicativa desde todas las asignaturas de dichos Grados, o implementar programas específicos que acompañen al alumnado durante su formación inicial. Otros estudios, ofrecen propuestas de mejora aplicables a mejorar la competencia comunicativa en el marco de las asignaturas que se imparten en las titulaciones. De acuerdo a Herrera et al. (2012), sería conveniente que el docente presente la rúbrica, se explique y debata previamente con los estudiantes, para lograr una mayor implicación de estos en las actividades que promueven estas habilidades. E incluso sería interesante consensuar y construir con el propio alumnado, una propuesta de rúbrica, lo cual podría contribuir a una mayor consciencia y reflexión por su parte, de las habilidades comunicativas que deberían dominarse exitosamente.

Según Berbey-Álvarez et al. (2017), los elementos gráficos presentes en los pósteres para exponer los resultados de una investigación, bien por medio de tablas o gráficas, son fundamentales para facilitar la comprensión y la retención del contenido y, por tanto, los resultados que se quieren comunicar de la investigación. Este aspecto parece que ha sido percibido positivamente por parte del alumnado, pues ha sido uno de los contenidos que se ha mejorado tras el desarrollo del T.PC.

Finalmente, conviene ser críticos con los resultados de esta investigación, que permiten proponer propuestas de mejora para próximos cursos. Así, se deben seguir incluyendo talleres formativos específicos sobre competencia comunicativa y también hacer un intento de integrarlas dentro del horario de las asignaturas, como contenido más presente, ofreciendo 
más oportunidades didácticas de que el alumnado pueda debatir, argumentar, presentar y sintetizar información.

\section{Agradecimientos}

Proyecto de Actuaciones Avaladas financiado por el Vicerrectorado de Innovación Docente de la Universidad de Cádiz (Sol-201800112449-tra).

\section{Referencias}

Albendín G., Arellano-López J. M., Vicente-Martorell J. J. (2019). Iniciación a la realización de pósteres científicos y desarrollo de habilidades comunicativas en alumnos de la asignatura de toxicología ambiental y salud pública. Revista de Toxicología, 36(1), 32.

Arquero J. L., González-González J.M. Hassall T., Joyce, J. (2012) Autoconcepto en capacidades de comunicación y aprensión: un estudio comparativo de los estudiantes en los nuevos grados del área de Ciencias Económicas y Empresariales. Revista de Contabilidad, 16(1), 24-31.

Asensio Pastor M. I. (2019) La lectura y la escritura académica en educación superior: el taller como estrategia didáctica. Psychology, Society, \& Education, 11(2), 205-219.

Balaguer Fàbregas M. C., Fuentes Loss M., y Palau Royo M. (2015) La competencia comunicativa oral en la formación inicial de maestros y maestras. Opción, 5, 15-32.

Berbey-Álvarez A., Álvarez H., Castillo G., De la Torre Diez I. (2017) El póster científico: recurso de la docencia e investigación. IV Jornadas Iberoamericanas de Innovación Educativa En El Ámbito de Las TIC, 115-122. Las Palmas de Gran Canaria.

Betancourt R., Guevara L., Fuentes E. (2011) El taller como estrategia didáctica, sus fases y componentes para el desarrollo de un proceso de cualificación en tecnologias de la información y la comunicación (TIC) con docentes de lenguas extranjeras. Caracterización y retos. Universidad de la Salle. Facultad de Ciencias de la Educación.

Blanco J. M., Díaz J., García E., Pacheco E. (2017) Evaluación del Máster de Investigación en Cuidados de la Salud de la Universidad Complutense de Madrid. En M. I. Ugarte Gurrutxaga (Ed.), IX Jornadas de Profesorado de Centros Universitarios de Enfermería. "La Investigación en Enfermería” (pp. 124-137).

Crujeiras B., Alexaindre M. P. (2012) Participar en las prácticas científicas. Alambique, 72, 12 19.

De la Fuente Prieto J., Asensio Castañeda E., Smalec Mallloy I. A., Blanco Fernández, A. (2015) Autoevaluación y desarrollo de habilidades comunicativas en profesores universitarios mediante e-rúbricas y grabaciones. Revista de Docencia Universitaria, 13(1), 257-276.

Díaz-Barriga F. (2010) Los profesores ante las innovaciones curriculares. Revista Iberoamericana de Educación Superior, 1(1), 37-57.

Díaz M. R., y Muñoz A. (2013) Los murales y carteles como recurso didáctico para enseñar ciencias en Educación Primaria. Revista Eureka Sobre Enseñanza y Divulgación de Las Ciencias, 10(3), 468-479.

Domènech Casal J. (2019) Estrategias lingüísticas para el tránsito a la competencia científica. Hablar y escribir para pensar en el aula de ciencias. Revista Investigación en la Escuela, 97, 47-63. 
Domingo J., Gallego J. L., Rodríguez A. (2013) Percepción del profesorado sobre la competencia comunicativa en estudiantes de Magisterio. Perfiles Educativos, 35(142), 54 74.

González-Sanz J. D., Barquero-González A. (2012) Simulacro de congreso científico como entrenamiento en competencias comunicativas en enfermería. Revista Iberoamericana de Educación e Investigación en Enfermería, 2(4), 20-28.

González J. D., Barquero A., Feria D. J., León R., Martín R. (2012) La competencia comunicativa oral en la formación inicial de maestros y maestras. TESI, 13(3), 162175.

Gramaglia C., Cignetti L., Aressi G., Cadoche L. (2018) Exploración de las competencias para la comunicación oral de estudiantes de asignaturas electivas del programa de la carrera Medicina Veterinaria de la Facultad de Ciencias Veterinarias de la Universidad Nacional del Litoral. Revista de Docencia Universitaria, 16(2), 281-292.

Herrera M., Sales D. L., Ben T., González D. (2012) Enseñanza y evaluación de la competencia transversal de aptitud para la comunicación oral. Jornada de Innovación Docente-RIMA, 184-199.

Hodson D. (1994) Hacia un enfoque más crítico del trabajo de laboratorio. Enseñanza de Las Ciencias: Revista de Investigación y Experiencias Didácticas, 12(3), 299-313.

Ibermón F., Medina J. L. (2008) Metodología participativa en el aula universitaria. La participación del alumnado. En M. Martínez y S. Carrasco (Coords.). Propuestas para el cambio docente en la universidad. Pp: 91-122. Barcelona: Octaedro.

Jiménez-Tenorio N., Vicente J. J. (2018) I Congreso de estudiantes sobre Didáctica de las Ciencias Experimentales en la Universidad de Cádiz: experiencia educativa en el Grado de Maestros de Educación Primaria. IV Simposio Internacional de Enseñanza de las Ciencias.

Llorente I., Domènech X., Ruiz N., Selga I., Serra C. Domènech-Casal J. (2017) Un congreso científico en secundaria: articulando el aprendizaje basado en proyectos y la indagación científica. Investigación en la Escuela, 91, 72-89.

Marines M., Heredia N. G., Solís L., Mena A. (2014) Taller multidisciplinario para el desarrollo de competencias de comunicación lingüística de la investigación. Formación Universitaria, 7(2), 41-50.

Montejo M. (2016) La preparación y edición de artículos científicos en el proceso de formación del investigador. Pedagogía Universitaria, 21(1), 16-28.

Moreno I. (2004) La utilización de medios y recursos didácticos en el aula. Departamento de Didáctica y Organización Escolar Facultad de Educación, Universidad Complutense de Madrid.

Pedrinacci E., Caamaño A., Cañal P., De Pro A. (2012) 11 ideas clave. El desarrollo de la competencia científica (Graó). Barcelona.

Pompa López Y., Pérez López, I. A. (2018) El desarrollo de la comunicación, desde su condición de habilidad profesional, en estudiantes de carreras pedagógicas. Conrado, 14(61), 45-52.

Rodríguez-García A. M., Martínez-Heredia N. (2019) Conocimiento profesional del docente en educación superior: una confrontacion entre lo real y lo deseado. Revista Iberoamericana de Educación Superior, 10(29), 25-41. 
Rodríguez K., Vargas K. (2009) Análisis del experimento como recurso didáctico en los talleres de ciencias: el caso del museo de los niños de Costa Rica. Revista Electrónica “Actualidaes Investigativas En Educación," 9(1), 1-20.

Santos Guerra M.A. (1991) ¿Cómo evaluará los materiales? Cuadernos de Pedagogía, 194, 29-31.

UCA (2012) Memoria del Título de: Grado en Ciencias ambientales por la Universidad de Cádir:

UCA (2013) Memoria del Titulo: Graduado o Graduada en Educación Primaria por la Universidad de Cádir: 